\title{
Rea

\section{Evaluación del trabajo en equipo en emergencias obstétricas, ¿contamos con las herramientas necesarias?}

Artículo original: Onwochei DN, Halpern S, Balki M. Teamwork Assessment Tools in Obstetric Emergencies: A Systematic Review. Simul Healthc. 2017 Jun;12(3):165-76. (PubMed)

Ródenas Monteagudo MA, Gutiérrez Pérez O, Romero García E, Argente Navarro P.

Hospital Universitari i Politecnic La Fe, Valencia.

\section{Resumen}

Las emergencias obstétricas son un escenario que aparece con relativa frecuencia. En los últimos años, diferentes trabajos orientan a que muchos eventos adversos en obstetricia se producen por un pobre trabajo en equipo durante las situaciones de crisis. Existen varias herramientas que evalúan las habilidades no técnicas de los equipos en estas situaciones. El objetivo de este trabajo fue valorar si estas herramientas son realmente válidas en el contexto obstétrico.

\section{Introducción}

Las emergencias obstétricas son un escenario que aparece con relativa frecuencia. En los últimos años, diferentes trabajos orientan a que muchos eventos adversos en obstetricia se producen por un pobre trabajo en equipo durante las situaciones de crisis. Existen varias herramientas que evalúan las habilidades no técnicas de los equipos en estas situaciones. El objetivo de este trabajo fue valorar si estas herramientas son realmente válidas en el contexto obstétrico.

\section{Introducción}

Las emergencias obstétricas (hemorragia postparto, cesárea emergente, paro cardiorrespiratorio en gestante, etc.) son un escenario que acontece con relativa frecuencia. Aunque el porcentaje de pacientes obstétricas que requieren cuidados intensivos es relativamente bajo $(0,7-$
0,9\%), la incidencia de morbilidad severa materna parece estar aumentando en este grupo de pacientes. Los resultados de diferentes estudios realizados en la última década orientan a que muchos eventos adversos obstétricos se producen por un deficiente trabajo en equipo durante las situaciones de crisis (1).

La participación efectiva de un equipo multidisciplinar que incluye enfermería, obstetras, neonatólogos y anestesiólogos en estas situaciones, puede resultar determinante para el bienestar maternofetal (2).

Para conseguir este objetivo, es imprescindible que el equipo desarrolle habilidades no técnicas que incluyen: comunicación, liderazgo y conducta de rol. La simulación clínica $y$, concretamente, el "Crisis Resource Management" permite entrenar dichas competencias (3). 
Disponemos de diferentes herramientas para evaluar las habilidades no técnicas (4). Sin embargo, no existe un consenso claro sobre si dichas herramientas son válidas en este contexto obstétrico específico.

\section{Resumen}

Se lleva a cabo una revisión sistemática cualitativa con el objetivo de encontrar herramientas de evaluación de la efectividad del trabajo en equipo en el contexto de las emergencias obstétricas, analizando tanto sus fortalezas como sus debilidades.

Para ello, los autores realizan una búsqueda en Embase, Medline, PubMed, Web of Science, PsycINFO, CINAHL y Google Scholar de estudios prospectivos que evalúen habilidades no técnicas en equipos multidisciplinares, abarcando desde 1944 hasta enero de 2016. Se excluyen editoriales, artículos de opinión y estudios que evalúan competencias individuales.

Los datos recogidos en el análisis fueron: los objetivos del estudio, herramienta de medición utilizada y los ítems que valora la misma, los escenarios de estudio, número de evaluadores y tipo de entrenamiento del evaluador. Se identifican nueve herramientas (tabla 1).

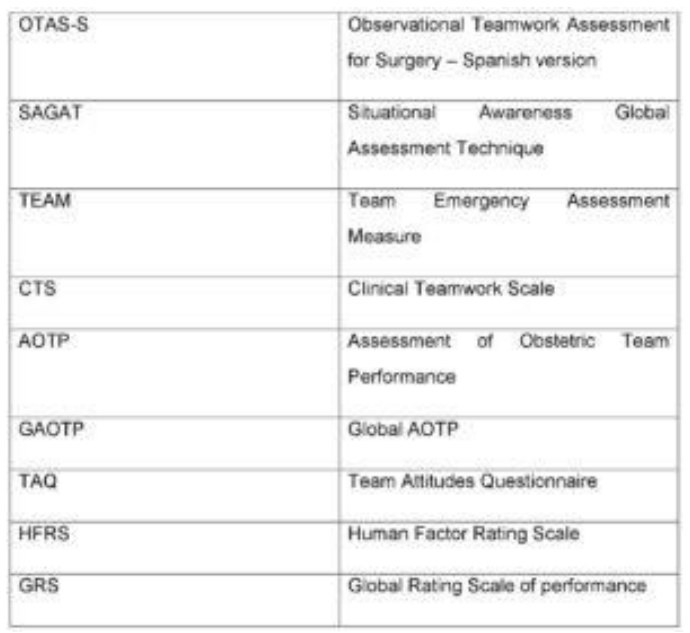

Tabla 1. Herramientas que valoran el trabajo en equipo en la simulación de escenarios de emergencias obstétricas.

Para dirigir la revisión se emplea la guía PRISMA, que juzga el riesgo de sesgos e incluye algunas medidas de calidad (número de evaluadores, si el equipo es multidisciplinar, si los escenarios de simulación son apropiados, si la herramienta fue usada en otras prácticas y si hay evidencia de mejores resultados clínicos gracias a su desarrollo). Se realizó un análisis descriptivo dada la naturaleza de los datos.

De los 15.333 estudios quedaron finalmente seleccionados 13 trabajos. Las habilidades no técnicas evaluadas en la mayoría son comunicación, consciencia situacional, roles de liderazgo y responsabilidad. No obstante, las escalas de puntuación fueron muy heterogéneas.

En la totalidad de los estudios evaluaron a los equipos en el contexto de escenarios de simulación obstétrica, sin embargo, la composición del equipo multidisciplinar no era homogénea. Los anestesiólogos solo participaban en los estudios que aparecen en la tabla 2.

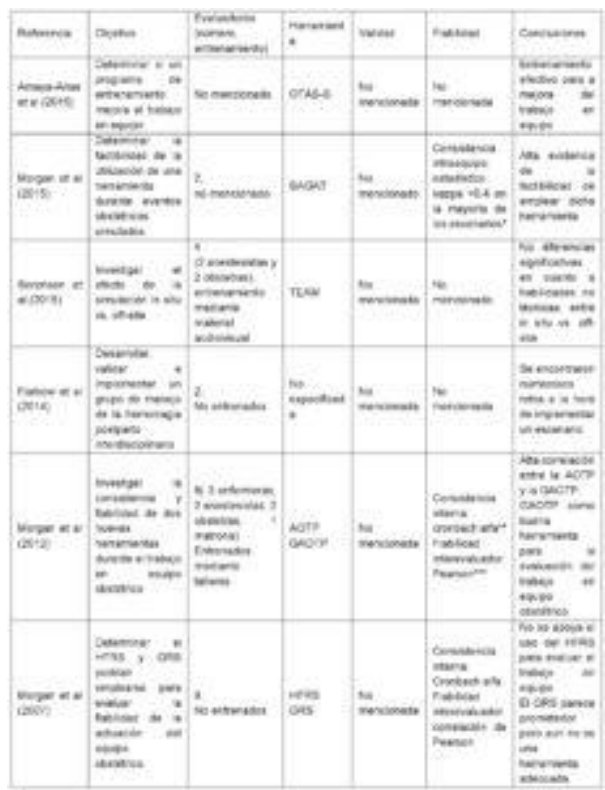

Tabla 2. Trabajos que incluyen anestesiólogos en la simulación de escenarios de emergencias obstétricas. 
* https://www.fisterra.com/mbe/investig a/kappa/kappa2.pdf

** https://www.uv.es/ friasnav/AlfaCro nbach.pdf

*** http://www.statisticshowto.com/pro bability-and-statistics/correlationcoefficient-formula/

Tampoco existe homogeneidad en cuanto a número, categoría profesional (enfermero, obstetra, anestesiólogo...) ni experiencia de los evaluadores que participan en la valoración de las herramientas. En 8 de los 13 trabajos se aseguraba el entrenamiento de los evaluadores en simulación clínica. El método de entrenamiento no se especifica en 3 de ellos y en el resto de los casos, variaba desde talleres prácticos al uso de vídeos. Es conveniente destacar que todos los trabajos a excepción de uno utilizaron vídeos como método de valoración del trabajo en equipo.

Las herramientas de valoración se analizan desde la perspectiva de validez ( 1 caso) de la confiabilidad (5 estudios) o ambas (un caso). Cabe mencionar que ninguno de los estudios llevó a cabo la evaluación de la calidad de las herramientas en cuanto a los resultados obtenidos en los pacientes.

\section{Conclusiones}

La simulación clínica ha demostrado ser una herramienta útil para el entrenamiento del trabajo en equipo y mejora las habilidades no técnicas. Sin embargo, actualmente no existe consenso sobre la herramienta más adecuada para valorar estos aspectos. Los requisitos necesarios para interpretar si un test es adecuado o no son poseer máxima fiabilidad y validez. En la presente revisión sólo un trabajo utilizó un test que valoraba ambas medidas (el 12).
La fiabilidad de una herramienta describe cómo de reproducible es en dos situaciones separadas por un cierto tiempo y la homogeneidad en las valoraciones del evaluador. Se cuantifica generalmente con IC, estadístico $\mathrm{K}$ y coeficiente de correlación de Pearson. La validez describe si la herramienta está realmente midiendo aquello por lo que se creó, incluye validez de contenido (si incluye todos los ítems importantes), validez de construcción (si proporciona mejorías clínicas reales tras utilizarla) y validez aparente (si las personas consideran que el test es útil para su trabajo).

De las herramientas identificadas las que resultaron tener mayor fuerza en su medición fueron la Clinical Teamwork Scale (CTS), la Global Assessment of Obstetric Team Performance (GAOTP) y la Global Rating Scale of Performance (GRS), ya que son las que tienen los mejores ítems valorados, facilidad de uso y fiabilidad en las mediciones realizadas.

Un aspecto importante para detectar si estas herramientas permiten evaluar dichas habilidades es la homogeneidad de los evaluadores en cuanto al número y la forma de entrenamiento que reciben. Se estima que debería haber más de dos evaluadores, para dotarle de fiabilidad.

Hay que considerar que no se valora la totalidad de competencias que pueden repercutir en la mejora de la seguridad del paciente: entre otras, claridad de rol y toma de decisiones. Además, ninguna de estas destrezas (comunicación, liderazgo y conciencia situacional), son exclusivas de las emergencias obstétricas y pueden aplicarse de la misma forma a equipos de trabajo de todas las especialidades médicas. 
Restringir la revisión a trabajos en ámbito de las emergencias obstétricas puede suponer una limitación en sí misma.

La herramienta ideal debería también evaluar la mejora de los resultados clínicos una vez trasladadas a situaciones cotidianas las habilidades aprendidas (5).

Podemos concluir que son necesarios más estudios que contemplen la evaluación de las habilidades no técnicas necesarias para un eficiente trabajo en equipo en otras subespecialidades médicas y que los resultados tras el entrenamiento de dichas competencias se reflejen en la práctica clínica diaria mejorando los resultados en los pacientes.

\section{Bibliografía}

1. Guise J-M, Segel S. Teamwork in obstetric critical care. Best Pract Res Clin Obstet Gynaecol. 2008 Oct;22(5):937-51. (PubMed) (HTML) (PDF)

2. Deering S, Johnston LC, Colacchio K. Multidisciplinary teamwork and communication training. Semin Perinatol. 2011 Apr;35(2):89-96. (PubMed) (HTML)

3. Tregunno D, Pittini R, Haley M, Morgan PJ. Development and usability of a behavioural marking system for performance assessment of obstetrical teams. Qual Saf Health Care. 2009 Oct;18(5):393-6. (ubMed) (HTML)

4. Guise J-M, Deering SH, Kanki BG, Osterweil P, Li H, Mori M, et al. Validation of a tool to measure and promote clinical teamwork. Simul Healthc. 2008;3(4):217-23. (PubMed)

5. Sheen J-J, Lee C, Goffman D. The utility of bedside simulation for training in critical care obstetrics. Semin Perinatol. 2017 Nov 24. (PubMed) (HTML)

Correspondencia al autor

Miguel Ángel Ródenas Monteagudo miguelangelrodenasmonteagudo@gmail.com FEA Anestesia y Reanimación. Hospital Universitari $i$ Politecnic La Fe, Valencia.

Aceptado para blog en junio de 2018. 